\title{
DESIGN AND FIELD TUNING OF AN UPSTREAM CONTROLLED CANAL NETWORK SCADA ${ }^{\dagger}$
}

\author{
M. RIJO* \\ Departamento Engenharia Rural, Universidade de Évora, Apartado 94, 7002-554 Évora, Portugal
}

\begin{abstract}
This paper presents the design, implementation and field tuning of a SCADA of an irrigation canal network upstream controlled with AMIL gates and equipped with other Neyrpic devices. Besides the hydraulic system, the paper also presents the supervisory and control system and its remote terminal units. The developed and field-tuned manual and automatic controllers are also presented. The manual controllers - direct and gate position controllers and gate flow controllers - are defined for the main canal intakes in order to permit achievement of predefined flow values or daily flow schedules, and are also defined for a few gate-controlled canal orifices used to discharge the overflow to the drainage system. The automatic controllers - water depth controllers for a few gate-controlled canal orifices - are defined in order to automatically prevent canal overtopping.

The SCADA system also monitors the outflows at the main canals - the most important canal top side weirs, canal terminal weirs and automatic Neyrpic siphons. The flow equations used in the computing of flow in real time inside the developed controllers and the monitoring units were field tuned using collected data readings from a monostatic Doppler current meter. The tuning parameters are also defined and presented. Copyright (C) 2008 John Wiley \& Sons, Ltd.
\end{abstract}

KEY WORDS: irrigation canal; irrigation canal modernization; local upstream control; real-time supervisory control; SCADA; flow equations

Received 7 January 2007; Revised 2 July 2007; Accepted 3 July 2007

\section{RÉSUMÉ}

L'article présente un système de télégestion développé et calé sur le terrain pour un réseau de canaux d'irrigation à commande par l'amont avec des vannes AMIL et pourvu d'autres équipements Neyrpic (comme par exemple, des siphons automatiques et des modules à masque). A côté du système hydraulique, l'article présente le système de télésurveillance et de régulation et ses unités terminales. Les contrôleurs, manuels et automatiques, développés et calés sur le terrain sont aussi présentés. Les contrôleurs manuels - directs, de fonctionnement des vannes et de débit des vannes - sont définis pour les canaux principaux de façon à permettre des débits pré-définis ou des consignes journalières de débit, et sont aussi définis pour quelques exutoires vannés destinés à évacuer le trop plein vers le système de drainage. Les contrôleurs automatiques - profondeur de l'eau pour quelques exutoires vannés - sont définis de façon à prévoir automatiquement tout débordement.

Le système de télégestion pilote également les débits de sortie des canaux principaux - les déversoirs des canaux les plus importants, les déversoirs de fin de canaux, et les siphons automatiques Neyrpic. Les équations utilisées pour calculer les débits en temps réel dans les contrôleurs ou dans les unités de surveillance ont été calées sur le terrain à l'aide d'un moulinet monostatique Doppler. Les paramètres correspondants sont aussi précisés et présentés. Copyright (C) 2008 John Wiley \& Sons, Ltd.

* Correspondence to: M. Rijo, Departamento Engenharia Rural, Universidade de Évora, Apartado 94, 7002-554 Évora, Portugal. E-mail: rijo@uevora.pt

†Développement et calage sur le terrain d'un système de télégestion d'un réseau de canaux à commande par l'amont.

Copyright (C) 2008 John Wiley \& Sons, Ltd. 